\title{
On the Absolute Summability (A) of Infinite Series
}

By M. FeKete, University of Jerusalem.

Communicated by E. T. Copson.

(Received 14th April, 1932. Read 6th May, 1932.)

\section{$\S 1 . \quad$ A series}

(1)

$$
\sum_{n=0}^{\infty} a_{n}
$$

has been defined by J. M. Whittaker ${ }^{1}$ to be absolutely summable $(A)$, if

$$
\sum_{n=0}^{\infty} a_{n} x^{n}=f(x)
$$

is convergent in $(0 \leqslant x<1)$ and $f(x)$ is of bounded variation in $(0,1)$, i.e.

$$
\sum_{r=1}^{n}\left|f\left(x_{r}\right)-f\left(x_{r-1}\right)\right|<K \quad(0<K<\infty)
$$

for all subdivisions $\mathbf{0}=x_{0}<x_{1}<x_{2}<\ldots<x_{n}<1$.

As Dr Whittaker has shown, ${ }^{2}$ the absolute convergence of (1) implies its absolute summability $(A)$.

In this paper a new sufficient condition for the absolute summability $(A)$ of (1) is obtained. In $\S 2$, it is shown that (1) is absolutely summable $(A)$, if it is absolutely summable $(C, r)$ where $r$ is a positive integer.

The series (1) is said ${ }^{3}$ to be absolutely summable $(C, r)$, if the sequence $\left\{c_{n}^{(r)}\right\}$ of its Cesàro-sums

$$
c_{n}^{(i)}=\left[a_{0}\left(\begin{array}{c}
n+r \\
r
\end{array}\right)+a_{1}\left(\begin{array}{c}
n+r-1 \\
r
\end{array}\right)+\ldots+a_{n}\left(\begin{array}{c}
r \\
r
\end{array}\right)\right] /\left(\begin{array}{c}
n+r \\
r
\end{array}\right)
$$

of order $r,(r=1,2, \ldots)$, is of bounded variation, i.e., if

$$
\left|c_{1}^{(r)}-c_{0}^{(r)}\right|+\left|c_{2}^{(r)}-c_{1}^{(r)}\right|+\ldots+\left|c_{n}^{(r)}-c_{n-1}^{(r)}\right|<H
$$

where $n=1,2, \ldots ; 0<H<\infty$,

1 Proc. Edinburgh Mruth. Soc. (2), 2 (1930), 1-5, p. 1.

2 L.c. ${ }^{3}$, pp. 1, 2.

"Fekete, Math. és terméš. ért., 29 (1911), 719-726, p. 719. Similarly (1) is said to be absolutely summable $(H, r)$, if the sequence $\left\{h_{n}^{(r)}\right\}$ of its Hölder.sums $h_{n}^{(r)}=\left(h_{0}^{(r-1)}+\ldots+h_{n}^{(r-1)}\right) /(n+1)$ of order $r\left(r=1,2, \ldots ; h_{n}^{(0)}=a_{0}+\ldots+a_{n}\right)$ is of bounded variation; Fekete, Math. és termésì. ért., 32 (1914), 389-425, p. 392. 
or, in other words, the series

$$
\sum_{n=1}^{\infty} a_{n}^{(r)}
$$

where $a_{n}^{(r)}=c_{n}^{(r)}-c_{n-1}^{(r)}$, is absolutely convergent.

It is easy to prove ${ }^{1}$ that every absolutely convergent series is also absolutely summable $(C, r)$ (for any positive integral value of $r$ ). Hence the theorem formulated above includes Whittaker's result.

It is worth mentioning that in the theorem stated above the words "absolutely summable $(C, r)$ " can be replaced by the words " absolutely summable $(H, r)$,"3 since these absolute summabilities are equivalent. ${ }^{2}$

This shows the analogy between the theorem considered and those due to Frobenius and Holder, stating the existence of the Abel limit $\lim _{x \rightarrow 1-0} f(x)$, i.e., the summability $(A)$ of (1), provided that this series is summable $(H, 1)$ and $(H, r)(r \geqslant 2)$ respectively.

$\S 2$. The convergence of (4) involves evidently that of (2) when $0 \leqslant x<1$. Thus, to prove our theorem, it must be shown that, the absolute convergence of (4) being assumed, the function $f(x)$ on the right of (2) satisfies (3). Since this inequality is equivalent to the existence of

$$
\int_{0}^{1} f f^{\prime}(t) d t
$$

now, as is easily verified,

$$
f^{\prime}(t)=\sum_{n=1}^{\infty} n a_{n} t^{n-1}=(1-t)^{r} \sum_{n=1}^{\infty}\left(\begin{array}{c}
n+r \\
r
\end{array}\right) n a_{n}^{(r)} t^{n-1},
$$

when $0 \leqslant t<1$, it is sufficient to prove that

$$
\int_{0}^{1}(1-t)_{n=1}^{r} \sum_{n=1}^{\infty}\left(\begin{array}{c}
n+r \\
r
\end{array}\right) n: a_{n}^{(r)} \mid t^{n-1} d t \text { exists and is equal to } \sum_{n=1}^{\infty}\left|a_{n}^{(r)}\right|,
$$

provided that the series at the end converges. ${ }^{3}$

1 L.c. ${ }^{3}$, p. 721.

2 L.c. ${ }^{3}$, pp. 397, 398.

s Conversely, the existence of the integral on the left of (6) involves the absolute convergence of (4). For from the equality (7) follows the inequality

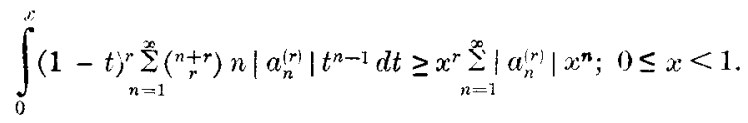


To show this, we derive by integration by parts

$$
\int_{0}^{x}(1-t)^{r} \sum_{n=1}^{\infty}\left(\begin{array}{c}
n+r \\
r
\end{array}\right) n\left|a_{n}^{(r)}\right| t^{n-1} d t=(1-x)^{r} \sum_{n=1}^{\infty}\left(\begin{array}{c}
n+r \\
r
\end{array}\right)\left|a_{n}^{(r)}\right| x^{n}
$$

$+(1-x)^{r-1} x \sum_{n=1}^{\infty}\left(\begin{array}{c}n+r \\ r-1\end{array}\right)\left|a_{n}^{(r)} \nmid x^{n}+\ldots+x_{n=1}^{r} \sum_{n=1}^{\infty}\left(\begin{array}{c}n+r \\ 0\end{array}\right)\right| a_{n}^{(r)} \mid x^{n} ; \quad 0 \leqslant x<1$.

Now, the absolute convergence of (4) being supposed, the last term of the foregoing sum tends, by Abel's theorem, to $\sum_{n=1}^{\infty}\left|a_{n}^{(r)}\right|$, when $x \rightarrow 1-0$, while under the same conditions its other terms, by a theorem ${ }^{1}$ of Cesàro, approach 0 ; this completes the proof.

§3. An infinite series may be absolutely summable $(A)$ without being absolutely summable $(C, r)$, of any order $r$. Let

$$
e^{\mathrm{i} /(1+x)}=\sum_{n=0}^{\infty} \alpha_{n} x^{n}
$$

The series on the right converges for $0 \leqslant x<1$ and the function $f(x)=e^{1 /(1+x)}$ satisfies (5), i.e., $\sum_{n=0}^{\infty} \alpha_{n}$ is absolutely summable $(A)$; but this series is not ${ }^{2}$ summable $(C, r)$ and hence, a fortiori, will not be absolutely summable $(C, r)$ for any value of $r$.

1 If $\alpha_{n}$ and $\beta_{n}$ are positive, then $\lim _{x \rightarrow 1-0} \sum_{n=0}^{\infty} \alpha_{n} x^{n} / \sum_{n=0}^{\infty} \beta_{n} x^{n}=\lim _{n \rightarrow \infty} \alpha_{n} / \beta_{n}$, provided that $\sum_{n=0}^{\infty} \alpha_{n} x^{n}, \sum_{n=0}^{\infty} \beta_{n} x^{n}$ converge in $(0 \leq x<1)$, the limit on the right exists and $\lim _{x \rightarrow 1-0} \sum_{n=0}^{\infty} \beta_{n} x^{n}=+\infty$. (Cf. Hobson, Theory of Functions of a Real Variable, 2 (1926), 175 177.) Apply Cesàro's theorem for $\alpha_{n}=\sum_{\nu=1}^{n}\left(\begin{array}{c}\nu+r \\ r-\mu\end{array}\right)\left|a_{\nu}^{(r)}\right|, \beta_{n}=\left(\begin{array}{c}n+r \\ r-\mu\end{array}\right)$.

2 This example is due to H. Bohr. Of. Landau, Darstellung u. Beyrundiung einiger neuerer Ergebnisse der Funktionentheorie (1929), \$7, p, 51. 